$15-1 / 2(2012)$

Varia

Sébastien Tran

\title{
Le pilotage des entreprises étendues: le rôle du SI dans le dispositif de gestion
}

\begin{abstract}
Avertissement
Le contenu de ce site relève de la législation française sur la propriété intellectuelle et est la propriété exclusive de l'éditeur.

Les œuvres figurant sur ce site peuvent être consultées et reproduites sur un support papier ou numérique sous réserve qu'elles soient strictement réservées à un usage soit personnel, soit scientifique ou pédagogique excluant toute exploitation commerciale. La reproduction devra obligatoirement mentionner l'éditeur, le nom de la revue, l'auteur et la référence du document.

Toute autre reproduction est interdite sauf accord préalable de l'éditeur, en dehors des cas prévus par la législation en vigueur en France.
\end{abstract}

\section{revues.org}

Revues.org est un portail de revues en sciences humaines et sociales développé par le Cléo, Centre pour l'édition électronique ouverte (CNRS, EHESS, UP, UAPV).

Référence électronique

Sébastien Tran, « Le pilotage des entreprises étendues : le rôle du SI dans le dispositif de gestion », Finance Contrôle Stratégie [En ligne], 15-1/2 | 2012, mis en ligne le 02 août 2012, consulté le 25 novembre 2012. URL : http://fcs.revues.org/99

Éditeur : Association FCS

http://fcs.revues.org

http://www.revues.org

Document accessible en ligne sur :

http://fcs.revues.org/99

Document généré automatiquement le 25 novembre 2012

Tous droits réservés 


\section{Sébastien Tran}

\section{Le pilotage des entreprises étendues : le rôle du SI dans le dispositif de gestion}

\section{Introduction}

L'organisation industrielle s'est profondément transformée pour répondre aux nouveaux impératifs des marchés (personnalisation de l'offre, réduction du time to market...) et aux tendances économiques structurelles (globalisation, chrono-compétition, dérégulation...). Dans ce contexte, les entreprises se sont elles aussi reconfigurées avec notamment l'apparition de formes hybrides (entreprise réseau, entreprise virtuelle, entreprise étendue) se situant entre le marché et la hiérarchie et s'insérant dans des réseaux de plus en plus ouverts et larges (Richardson, 1972 ; Williamson, 1985 ; Thorelli, 1986 ; Konsynski, 1993 ; Jarillo, 1993 ; De Vaujany, 2010). L'entreprise étendue est ainsi devenue une forme organisationnelle à part entière et a fait l'objet de nombreuses recherches. La plupart des définitions dans la littérature apparentent le concept d'entreprise étendue à des relations avec des partenaires externes, une porosité des frontières organisationnelles et des modalités de coordination appropriées (Hagel, 1996). Nous proposons de retenir la définition suivante inspirée de Naulleau et Guth qui ont beaucoup travaillé sur le secteur automobile, mais dans un périmètre un peu plus restreint que celui proposé par les auteurs : «ensemble d'entités coordonnées de manière hiérarchique ou quasi-hiérarchique, alignées stratégiquement et économiquement à différents stades de la chaîne de valeur d'une activité donnée et intégrées par des systèmes avancés d'information, de communication et de logistique » (2000, p. 32).

Cette définition de l'entreprise étendue met l'accent sur l'élaboration d'une stratégie et son pilotage, notamment l'alignement stratégique de multiples entités et la mesure de la performance conformément au plan de progrès. Les systèmes de pilotage doivent donc assurer le déploiement de la stratégie dans l'ensemble de l'organisation en s'appuyant de plus en plus sur des outils de gestion qui sont définis comme « un ensemble d'objets intégrés de façon systématique et codifiée dans une logique fonctionnelle ou toute autre logique d'acteur, et respectant un certain nombre de règles de gestion » (De Vaujany, 2005). On observe la prolifération des outils de gestion depuis le XIX ${ }^{\text {ème }}$ siècle dans la plupart des organisations (Lorino et Teulier, 2005). Toutefois, on trouve peu d'outils de gestion répondant vraiment aux spécificités des entreprises étendues ou se revendiquant comme permettant de les piloter. Les différents outils de gestion et de pilotage (BSC, méthode $\mathrm{ABC}, \mathrm{TQM}^{1} \ldots$...) ne sont pas ou très peu compatibles les uns avec les autres, et, au-delà des normes de type ISO 9000, peu d'outils sont à même de proposer un pilotage de l'organisation dans sa globalité en intégrant toutes les entités.

La définition de l'entreprise étendue souligne également le rôle des Systèmes d'Information². Quelques travaux ont également montré leur importance dans la création de valeur à partir d'un alignement de la dimension stratégique, organisationnelle et technologique (Henderson et Venkatraman, 1993). Chaque évolution dans l'histoire des entreprises s'est accompagnée d'une configuration organisationnelle et de dispositifs de gestion spécifiques. Au même titre que le contrôle de gestion représente une instrumentation spécifique pour l'émergence de l'entreprise multi-divisionnelle (Chandler, 1962) qui constitue une forme d'entreprise étendue, les TIC procurent de nombreuses possibilités pour instrumenter les nouvelles modalités organisationnelles (Detchessahar, 1997 ; Besson et Rowe, 2011). Certaines configurations comme les firmes réseaux ou les firmes $\mathrm{M}$ n'échappent pas à cette logique car elles ne peuvent fonctionner que si elles sont capables de traiter des informations entre les unités dans une logique multidimensionnelle (Markus, 2010). Actuellement, les SI représentent un élément prépondérant du système de pilotage et de la stratégie des entreprises, notamment en soutenant des processus matérialisés par des outils de gestion informatisés (cf. tableau 1). 
Tableau 1 - Intégration des TIC dans le fonctionnement des entreprises en France

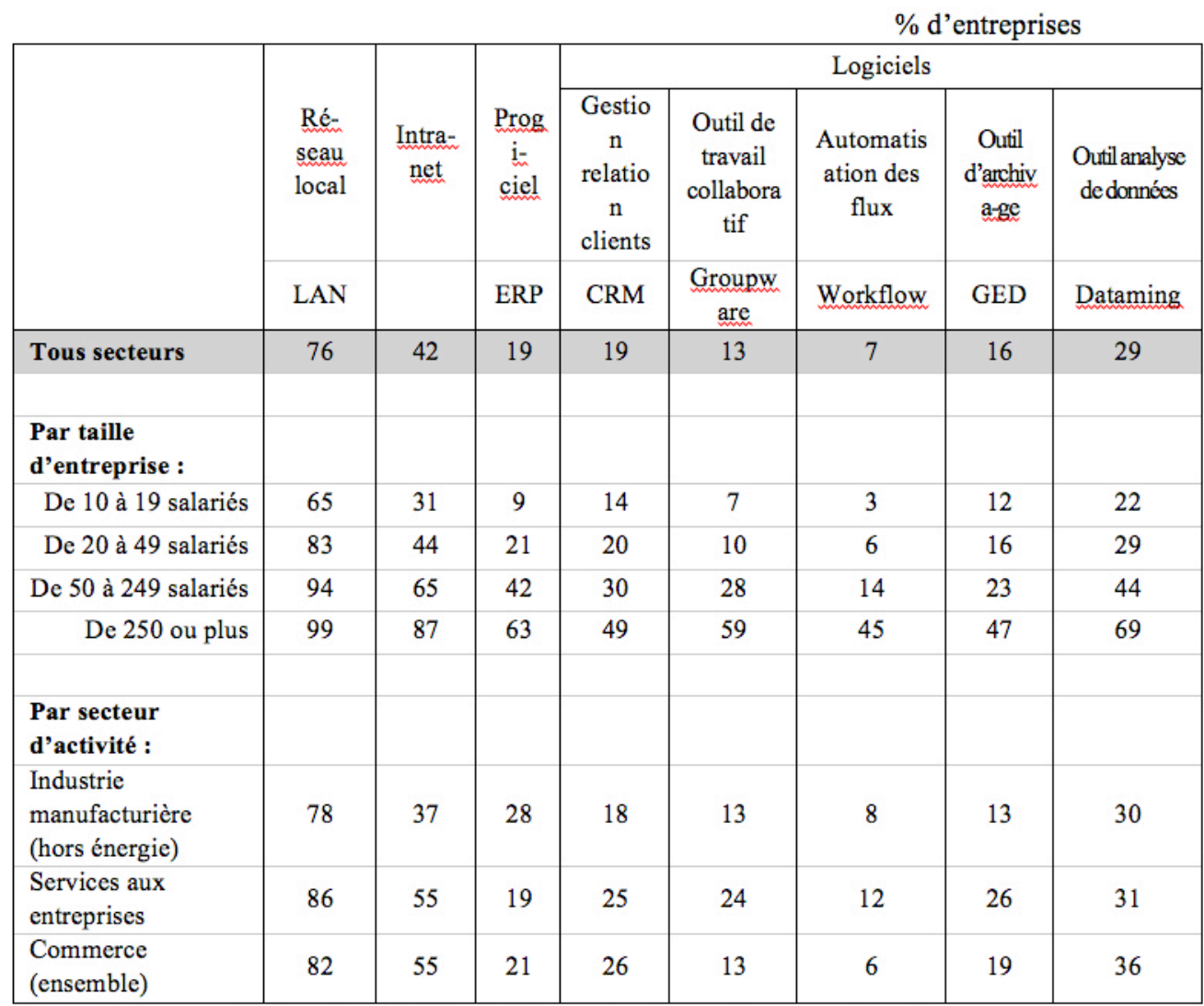

Champ : Entreprises de 10 salariés ou plus des secteurs marchands

Source : Insee-enquête TIC 2008

L'objet de cet article est donc de déterminer quelles sont les caractéristiques spécifiques et le statut des SI vis à vis des outils de gestion dans les entreprises étendues afin de permettre le pilotage de la stratégie. Il existe très peu de travaux sur le statut des SI par rapport aux outils de gestion alors qu'ils semblent jouer un rôle dans leur diffusion dans les organisations. La première partie de cet article sera ainsi consacrée aux apports des SI à l'instrumentation gestionnaire, notamment sur les dimensions du contrôle, de la coordination et du pilotage. Dans la seconde partie, nous présenterons notre méthodologie basée sur une étude de cas d'un équipementier automobile qui fonctionne comme une entreprise étendue et l'objet de gestion, les roadmaps de management, qui prend forme à partir d'un SI. Dans la dernière partie, nous analyserons les caractéristiques spécifiques de ce SI dans la diffusion de l'outil de gestion que sont les roadmaps de management et nous proposerons une discussion de notre cas en élargissant la réflexion à la place du SI en tant qu'élément structurant du dispositif de gestion et d'évolution de l'organisation.

\section{1-Quels apports du SI a l'instrumentation gestionnaire ?}

5 Les SI occupent une place importante dans les investissements des entreprises et leur diffusion relève désormais d'un choix stratégique. A partir d'une certaine taille, les problématiques organisationnelles au sein des entreprises (découpage des périmètres des unités, relations hiérarchiques, structure de l'organisation) ne peuvent être dissociées d'une réflexion sur l'urbanisation du SI et l'élaboration d'un schéma directeur ${ }^{3}$. Plusieurs travaux suggèrent que les SI auraient une dimension stratégique (Porter et Millar, 1985 ; Kettinger et al., 1994 ; Bernasconi, 1996) au sens d'être une ressource pouvant générer un avantage concurrentiel durable. On retrouve dans les caractéristiques des SI plusieurs dimensions relevant des outils de gestion : le contrôle, la coordination des différentes entités et le pilotage de l'organisation selon la stratégie définie. 


\subsection{Le contrôle}

Les SI s'insèrent dans la stratégie des firmes avec des objectifs récurrents de réduction des coûts et d'amélioration de la productivité. Les dimensions formelles de contrôle et d'évaluation sont devenues de plus en plus importantes (Ouchi, 1979) et se traduisent par des exigences en matière de reporting et de tableaux de bord. Les SI peuvent matérialiser ces outils dont les ERP représentent sans doute le meilleur exemple dans les organisations avec des applications comptables ou de contrôle de gestion. D'abord, on retrouve dans les SI une fonction de contrôle des processus au sens de l'agrégation des données des différentes entités dans une logique analytique grâce à l'interconnexion des applications et de langages communs (De Corbière, 2011). Les SI portent ainsi en eux un système de contrôle plus ou moins explicite (David et Pallez, 2001). Ensuite, les possibilités de formalisation offertes par les SI sont particulièrement intéressantes pour matérialiser les dispositifs de contrôle au sein des organisations (états comptables, tableaux de bord, indicateurs de reporting et de performance). Cette fonction s'avère encore plus importante dans les organisations étendues qui doivent consolider une grande masse d'informations issue de plusieurs entités. Dans le prolongement de cette logique, on peut ajouter que l'existence même du SI est encore plus liée à son utilisation aux niveaux opérationnels qui doivent alimenter ce dernier en données, et donc les outils de gestion associés. Le SI constitue dès lors le support informationnel des outils de gestion en permettant les échanges de flux d'informations entre les entités et les remontées vers la direction de l'entreprise soumise à des impératifs de diffusion de données consolidées, notamment pour les différentes parties prenantes (actionnaires, salariés, clients...).

Au-delà du système de contrôle, la mise en place d'un SI est concomitante à la création d'une grammaire technique avec un processus de normalisation des langages et l'automatisation du traitement des données (EDI, ERP, workflow...) (De Corbière, 2011). On voit alors se dessiner un processus informationnel qui permet de diminuer les coûts de coordination. Cette normalisation électronique a souvent été nécessaire dans l'intégration logistique de différents acteurs au sein d'une chaîne de valeur. Ces évolutions questionnent la nature même des relations entre les entités, d'autant plus lorsque ces dernières sont externes aux frontières de la firme (clients, fournisseurs, administrations...) ou lorsqu'elles possèdent un degré d'autonomie important. Les effets de l'implantation des SI au sein des organisations sont d'ailleurs très ambigus en matière de centralisation ou décentralisation. Les SI possèdent en effet la particularité de pouvoir supporter à la fois des organisations souples telles que les entreprises étendues ou en réseau, et des structures au contraire très formelles et hiérarchisées (Reix, 1999 ; Benghozi, 2001). Le SI peut donc favoriser la mise en place de structures quasihiérarchiques en étant l'infrastructure informationnelle entre les entités et la firme pivot dans une structure de type réseau.

\subsection{La coordination des entités}

Les organisations étendues doivent faire face à des problèmes de coordination plus complexes en raison d'un nombre important d'entités et d'une dimension spatiale et temporelle extensive et variable au fur et à mesure des décisions stratégiques (alliances, acquisitions, cessions, quasi-intégration...). La cohérence des décisions multiples repose alors sur des mécanismes de coordination où la communication d'information est primordiale, ce qui explique l'adoption rapide des SI dans les grandes organisations d'un point de vue historique. Cette logique a été élargie depuis plusieurs années aux relations externes de type clients-fournisseurs avec une imbrication de plus en plus forte des SI des différents acteurs dans la chaîne de valeur pour aboutir à l'apparition du concept de SI interorganisationnels (Barett et Konsynski, 1982 ; De Corbière, 2011). Comme nous l'avons vu précédemment, la normalisation des échanges de données (protocoles, format des données...) a contribué à la mise en place d'une coordination interne et en dehors du périmètre de l'entreprise. Les travaux s'accordent ainsi à présenter les SI déployés dans les relations interentreprises et intraentreprises comme des facilitateurs de la coordination entre les différents acteurs et comme des réducteurs des coûts de transaction (Clemons et Row, 1991 ; Argyres, 1999 ; Amabile et Gadille, 2006). 
La notion de modélisation de l'organisation (DiMaggio et Powell, 1983) est également présente dans le concept de SI car il doit permettre une structuration des données dans une logique organisationnelle définie selon la mise en adéquation de ressources et d'objectifs. Certains SI permettent de cartographier les entités de l'organisation et de les coordonner dans une logique d'action organisée afin d'atteindre les objectifs fixés au préalable par les outils et techniques de gestion. L'alignement des différents sites ou groupes de travail est souvent une condition essentielle dans l'atteinte des objectifs qui nécessite une cognition distribuée avec des SI comme support (Rasmussen et al., 1991). L'une des autres difficultés dans les organisations étendues est de savoir quelles sont les ressources et niveaux de performance des différentes entités, d'autant plus lorsqu'elles sont réparties d'un point de vue géographique et qu'elles possèdent un certain degré d'autonomie. Enfin, les SI peuvent faire évoluer le découpage des rôles des entités dans une logique projet en faisant état du niveau de chacune au moment de la prise de décision. Les SI servent de support aux outils de gestion visant à modéliser les ressources disponibles dans chacune d'entre elles. Il est ainsi parfois difficile pour une organisation de connaître le fonctionnement réel de ses propres entités. En ce sens, les outils de gestion permettent une investigation du fonctionnement organisationnel (Moisdon, 1997) et peuvent profiter des propriétés des SI pour affiner la modélisation des ressources disponibles dans les différentes entités de l'organisation en les obligeant à générer de l'information sur une base commune.

\subsection{Le pilotage et la stratégie}

Les SI peuvent être analysés comme un système de pilotage qui explicite et réorganise les relations et activités de travail entre les différentes entités. Cela positionne le SI comme une interface entre le système de pilotage, qui conçoit, organise le système de représentations (les objectifs, les orientations, les projets de l'entreprise) et décide, et le système opérant, opérationnel, qui conduit les actions quotidiennes (Le Moigne, 1977). Le SI doit être l'infrastructure qui permet des actions de commande, de régulation et de prise de décisions entre le système décisionnel et le système opérationnel. On retrouve une approche classique des SI nommée Decision Support System (DSS), qui les positionne comme un processus de résolution de problèmes et de prises de décision pour les acteurs à partir du traitement des informations, voire même allant jusque la création de métadonnées. Le fonctionnement des organisations repose également sur des boucles de rétroaction entre la direction et les entités. Ces interactions supposent alors un système de communication dans un environnement où la réactivité constitue désormais un facteur clé de succès. Elles s'appuient de plus en plus sur le SI pour les échanges formels et visent à réguler les écarts constatés, même si les relations informelles demeurent nécessaires pour enclencher des actions de régulations.

11 Nous savons depuis les travaux de Moisdon et al. (1997) que les outils de gestion ont un rôle important de conformation visant notamment à normer les comportements des acteurs. Les SI participent pleinement à ce processus via la création d'une représentation commune pour l'ensemble des acteurs, même si ces derniers peuvent ne pas la partager. En ce sens, le SI est porteur d'une philosophie gestionnaire et traduit des choix managériaux et stratégiques (interopérabilité des systèmes, logiciels libres vs logiciels propriétaires, gestion des droits d'accès...). Les informations diffusées par le SI vont conduire les acteurs à adopter certains comportements (parfois de contournement) ou à mettre en application certaines décisions pour atteindre les résultats escomptés. L'entreprise est un ensemble de systèmes sociaux, dont la régulation s'opère par des mécanismes de jeux à travers lesquels les calculs rationnels stratégiques des acteurs se trouvent intégrés en fonction d'un modèle structuré dont le SI constitue le support. Le SI représente alors un ensemble d'acteurs sociaux qui mémorisent et transforment des représentations via des technologies de l'information et des modes opératoires induits. Il va donc structurer les comportements des acteurs présents dans l'organisation et devenir ainsi pour les décideurs un outil de pilotage.

12 Cette première partie constitue une base pour un cadre théorique intégrateur en montrant que les SI constituent plus qu'un simple substrat technique aux outils de gestion. Même si la dimension de contrôle est sans doute la plus répandue parmi les SI actuellement, les dimensions 
de contrôle et de pilotage de stratégie peuvent s'avérer pertinentes selon les configurations organisationnelles car elles peuvent accentuer le rôle de certains outils de gestion jusqu'alors peu pourvu en matière d'infrastructure technique, voire même contribuer à modifier la philosophie sous jacente. Enfin, peu de travaux se sont intéressés à la prise en compte de l'interaction outils de gestion/SI alors qu'elle permet d'expliquer les transformations organisationnelles. C'est ce que nous chercherons à montrer dans la partie suivante à partir de notre étude de cas.

\section{2- Méthodologie et présentation de l'outil de gestion : les roadmaps de management}

Dans le cadre de notre recherche et de notre problématique de départ, nous avons décidé d'opter pour une méthodologie de type qualitative car elle nous semble plus appropriée à notre questionnement de recherche. Nous nous appuierons donc sur une étude de cas d'un équipementier automobile qui a mis en place un outil de gestion créé par un cabinet de conseil, les roadmaps de management, avec comme support de diffusion dans l'organisation un SI nommé Matrix. Dans ce contexte, l'outil de gestion est au départ dissocié du SI car conçu de manière indépendante. L'objectif de cette recherche terrain est d'identifier les propriétés émergentes complexes de deux objets qui sont interdépendants (les SI et les outils de gestion) et non de mettre à l'épreuve un objet théorique. Dans ce cadre, Valeo est un cas représentatif : c'est un groupe industriel indépendant et international qui se classe parmi les premiers équipementiers automobiles mondiaux et qui est structuré à partir de 10 branches ${ }^{4}$. La Direction Générale doit ainsi piloter un ensemble d'entités n'ayant pas les mêmes métiers mais partageant les mêmes exigences, notamment la production de biens de qualité à des coûts économiquement acceptables dans des délais raisonnables. Ce pilotage des entités par la Direction Générale du groupe est d'autant plus ardu que le groupe dispose d'entités sur les cinq continents.

La méthodologie de recherche

Nous avons réalisé cette recherche à partir d'une étude de cas définit comme « la recherche d'un phénomène contemporain dans son contexte réel et particulièrement quand les frontières entre le phénomène et le contexte ne sont pas clairement tracées » (Yin, 2003, p. 13). Cette opportunité d'étude de cas unique (Eisendhart, 1989) s'inscrit dans le cadre d'un projet ANR qui s'est terminé fin 2010. Le recueil de données s'appuie sur une observation participante (David, 1999) au sein du cabinet MNM consulting de décembre 2007 à juin 2010, à raison d'une présence sur le terrain de deux jours par mois (soit au sein du cabinet de conseil, soit en extérieur pour la réalisation d'entretiens, de recherche d'information, de participation à des conférences professionnelles...). Nous avons une étude de cas longitudinale approfondie sur 3 ans ce qui est peu courant pour appréhender les situations de transformations organisationnelles (Besson et Rowe, 2011). Cette étude de cas s'inscrit donc sur une durée significative, ce qui permet au chercheur de construire une compréhension en profondeur de l'outil de gestion et de sa médiatisation au sein d'un SI. MNM consulting est en charge de la mise en place d'un outil de pilotage des plans de progrès chez l'équipementier automobile Valeo, à savoir les roadmaps de management.

Le cabinet de conseil représente un endroit privilégié d'observation en matière d'accompagnement de l'équipementier dans la mise en place des roadmaps de management sur la plateforme informatique Matrix. Notre recherche utilise ainsi les données recueillies durant cette période pour décrire, analyser et comprendre le rôle du SI dans la mise en place d'un outil de gestion au sein d'une organisation étendue. MNM consulting est composé de 14 personnes et possède la particularité de faire du conseil pour les organisations tout en ayant une expertise 
d'ingénierie informatique (équipe de plusieurs développeurs en interne) et un département R\&D. Nous avons eu à deux reprises une présentation de la plateforme en situation d'utilisateur et nous avons participé à une étude de cas sous forme de jeu de simulation pour comprendre le fonctionnement des roadmaps. Nous avons également pu échanger régulièrement sur la conception et l'évolution du SI avec les développeurs, les consultants et le dirigeant de MNM Consulting.

Au niveau des données primaires, nous avons créé et exploité plusieurs verbatims à partir des réunions et des entretiens réalisés avec les consultants du cabinet MNM (cf. annexe 1) : le directeur, le formateur, les consultants, les ingénieurs R\&D et un doctorant. Nous avons retranscrit les entretiens et réalisé une analyse thématique par inférence afin de faire ressortir les thèmes représentatifs du rôle joué par le SI. En plus de nos entretiens chez MNM consulting, nous avons eu accès aux verbatims de 38 entretiens réalisés par deux doctorants (dont un en convention CIFRE au sein du cabinet de conseil) auprès de responsables (rédacteurs de roadmaps, auditeurs, responsables d'axes et ingénieurs opérationnels) situés dans différents services chez l'équipementier. Enfin, nous avons pu échanger à quelques reprises avec le DSI de Valeo et nous avons réalisé un entretien semi-directif avec le Group Office Information System Director de Valeo, notamment sur les fonctionnalités en 2.0 du SI.

Concernant les données secondaires, nous nous sommes appuyés pour illustrer les entretiens sur les documents édités par le cabinet de conseil et l'équipementier (rapports, notes de synthèse, comptes-rendus de réunions, Intranet du cabinet de conseil auquel nous avons eu accès, site Internet institutionnel, nombreux exemples de roadmaps de management en format Excel). Une fois les données primaires et secondaires collectées, nous avons réalisé une triangulation pour aboutir à un corpus de données fiables (Eisendhart, 1989) pour traiter notre question de recherche.

\subsection{Présentation du cas Valeo}

Valeo est représentative de notre définition des entreprises étendues. C'est une entreprise industrielle multibranches qui dispose de plus de 30 centres de production dans le monde, ce qui représente plus de 70000 salariés répartis dans plus de 25 pays différents. Par ailleurs, son périmètre évolue en permanence au gré des rachats, cessions et partenariats avec des entreprises de la filière automobile. Le fonctionnement interne ancré dans la culture de l'entreprise est basé sur une formalisation accentuée issue de la culture ingénieurs et sur un principe de décentralisation avec une organisation multisites. Les entités opérationnelles sont autonomes pour mettre en œuvre la stratégie du groupe mais elles doivent respecter certaines pratiques et standards définis et formalisés par la direction. L'entreprise comporte plusieurs branches industrielles et le siège doit assurer le pilotage de l'ensemble avec un système de management approprié qui doit prendre en compte la distance géographique et culturelle des sites. Les enjeux se situent clairement sur un contrôle de niveau de maturité des sites, une mesure fiable de la progression au niveau des réseaux fonctionnels et la diffusion des meilleures pratiques car l'environnement de l'entreprise est très fortement concurrentiel. Par ailleurs, les prix, la qualité, les coûts et l'innovation sont de véritables facteurs clés de succès renforçant dès lors les enjeux d'alignement stratégique des sites. Il devient donc nécessaire d'adapter les outils de pilotage afin qu'ils puissent intégrer les problématiques de développement du progrès fonctionnel. 
Figure 1 - Architecture organisationnelle de Valeo

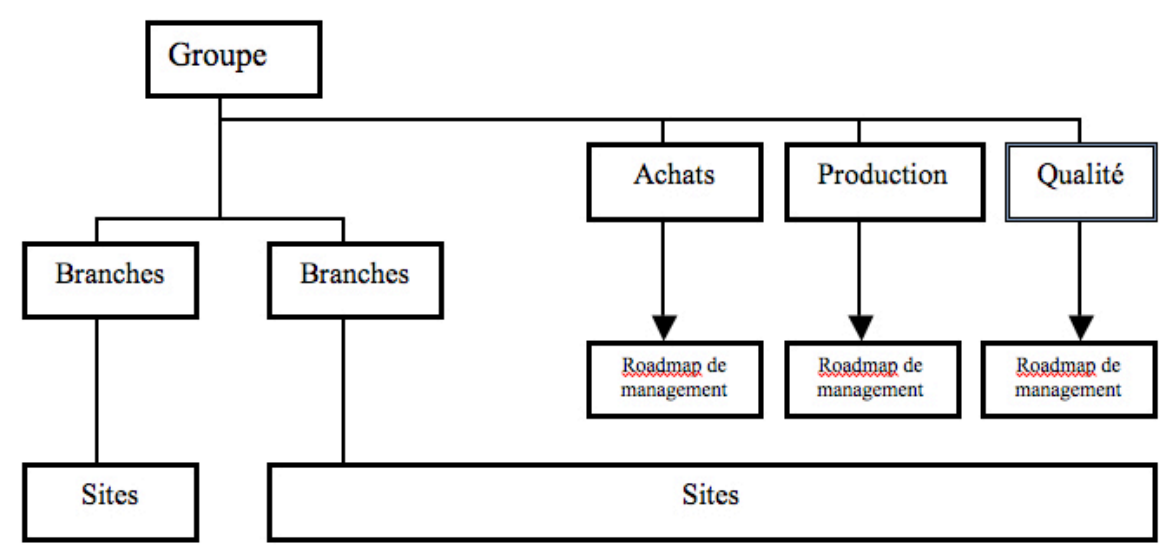

Source : MNM/Valeo

L'entreprise s'appuyait auparavant sur un système d'audit qualité traditionnel de type ISO mais ce dernier a rapidement montré ses limites en termes de pilotage, d'évaluation et d'alignement des sites au niveau de la direction. L'opérationnalisation de la stratégie doit se faire de manière régulière pour atteindre les résultats fixés par la direction de l'entreprise. Par ailleurs, les dirigeants souhaitent un retour des niveaux opérationnels afin également de mieux les impliquer dans la réalisation de la stratégie et de faire accepter cette dernière. Avec l'aide de MNM consulting, Valeo a décidé de déployer un dispositif de pilotage du progrès avec une méthode baptisée roadmaps de management et déposée sous la marque 5 Steps ${ }^{5}$. On retrouve ainsi le déploiement d'une méthode de gestion (5 Steps) et d'un outil de gestion (Roadmaps de management) dans une entreprise étendue en lieu et place d'un système de type ISO. Cette méthode repose sur un outil qui doit permettre un déploiement des standards, de l'auto-évaluation et un contrôle plus efficace de l'appropriation des bonnes pratiques par l'organisation.

Visuellement, les roadmaps de management sont des représentations graphiques sous forme de tableaux de bord bâtis sur deux dimensions structurantes et strictement identiques : la dimension évolution (niveau d'amélioration matérialisé par une échelle graduelle de 1 à 5 d'où le nom de la méthode Five Steps) et la dimension objets à améliorer ou leviers. Pour mieux appréhender les leviers, ceux-ci sont rattachés à des thèmes transversaux qui sont en fait une division de l'objet en différentes parties regroupant des éléments communs liés entre eux et porteurs de sens pour les acteurs. Les items représentent les différents éléments composant un thème. L'interaction entre un levier à piloter et le niveau d'amélioration permet de franchir des paliers qui correspondent à un état obtenu après la réalisation de différentes tâches. Il est important de noter que la justification de l'atteinte d'un niveau se traduit par des livrables adaptés à chaque roadmap de management (assertion, document, lien vers d'autres roadmaps). On est bien dans une culture de la preuve pour passer d'un niveau à un autre, ce qui peut s'apparenter à une traçabilité et un contrôle indirect. La roadmap (cf. figure 2) présente ainsi sous une forme de tableau croisé à 5 niveaux progressifs les meilleures pratiques et les objectifs élémentaires pour la réalisation du plan d'action stratégique. L'objectif est d'amener les responsables de roadmaps à faire une auto-évaluation en complétant eux-mêmes le niveau atteint et à le formaliser avec des délivrables, sachant qu'un audit est pratiqué une fois par an en se basant sur les roadmaps et leur niveau de maturité. 
Figure 2 - Architecture générique d'une roadmap de management

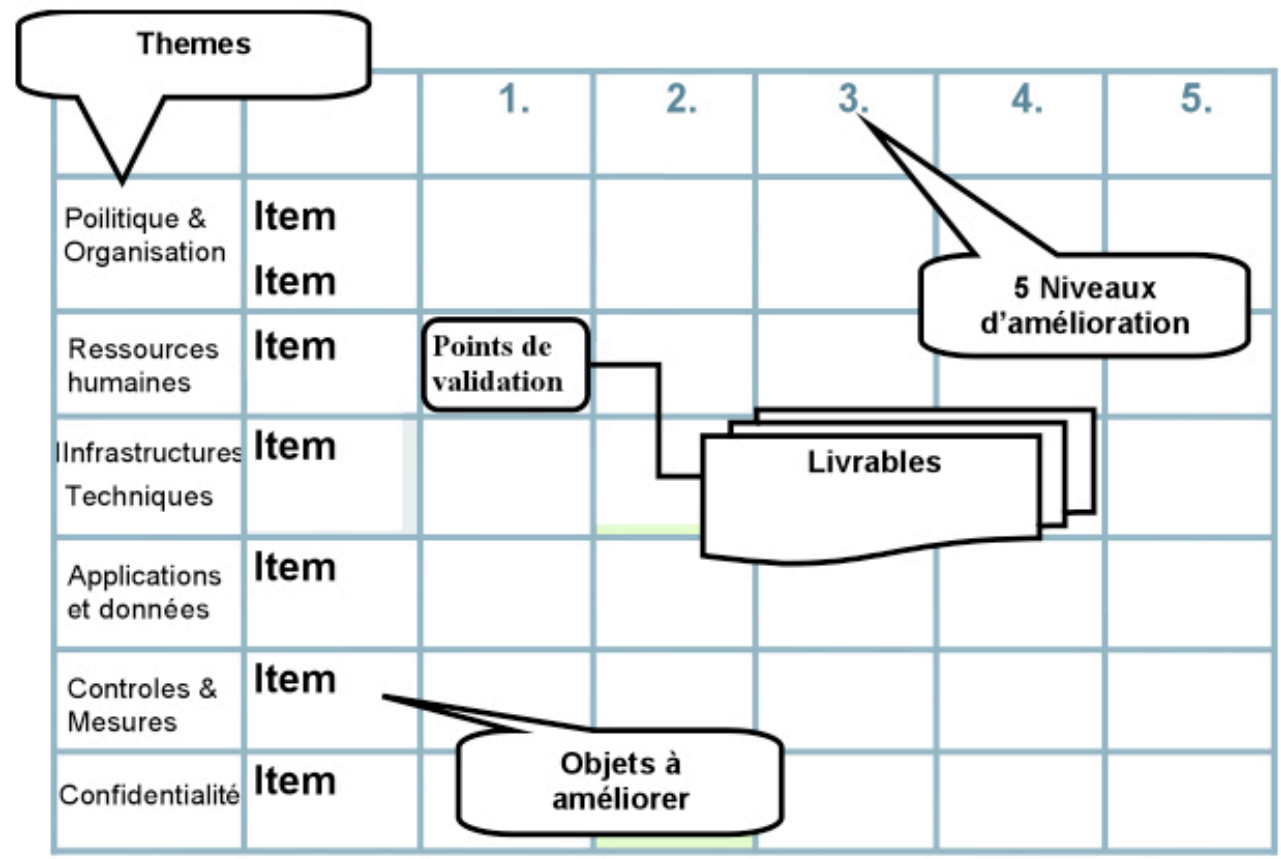

Source : MNM/Valeo

La méthode permet donc en théorie une progression continue et qualitative des différents sites qui sont dispersés géographiquement avec une optique d'alignement sur les meilleures pratiques, ainsi qu'une intégration des nouveaux sites avec des modalités efficaces d'apprentissage et d'évaluation. Les roadmaps s'apparentent alors à un système de structuration et de capitalisation des meilleures pratiques. L'un des enjeux pour l'équipementier est de généraliser les roadmaps à l'ensemble de l'organisation multisites à partir d'une plateforme commune. En effet, ces dernières ne font sens au niveau local et agrégé que si elles sont déployées dans l'ensemble de l'organisation puisque le niveau de performance s'établit également de manière relative entre les entités. La dimension systémique est donc encore plus présente dans les entreprises étendues et représente un véritable challenge pour les dirigeants. Il leur est donc apparu assez logique « d'informatiser leur outil de gestion » pour donner du sens aux indicateurs délivrés et comparer les niveaux de maturité atteints (on retrouve la logique de contrôle). La visibilité au niveau micro (entités) et méso économique (direction) n'est possible que si les informations sont traitées par un système centralisateur et rediffusées sous les formats appropriés aux différentes parties prenantes.

\subsection{La mediation des roadmaps de management via le SI}

La DSI de Valeo, après une recherche pendant plusieurs mois de solutions existantes sur le marché, est arrivée à la conclusion qu'il n'existait pas de SI dédiés répondant à la problématique de pilotage d'une organisation étendue dans une logique de progrès et d'autoassessment. Les SI s'avèrent inappropriés car ils sont soit trop centrés sur des processus métiers (comptabilité et gestion, supply chain...), soit peu modulables et contextualisables à des environnements spécifiques. Par ailleurs, les problématiques du coût, de l'interopérabilité et de la rapidité de déploiement apparaissent souvent comme prépondérantes dans le choix des SI. Ce fut notamment le cas chez Valeo, avec une contrainte supplémentaire issue d'une organisation structurée sur plusieurs sites répartis dans différentes zones géographiques. Les roadmaps de management, en tant qu'outil de gestion, ont donc été déployées à partir d'un SI déjà existant et implanté au sein de l'entreprise.

Dans le cadre de notre étude de Valeo, l'outil support de généralisation des roadmaps est Matrix (avec le support du progiciel SAP pour la consolidation des données) qui possède à la base un portefeuille d'applications en PLM $^{6}$ (gestion des achats, gestion documentaire, gestion de projet...). Certaines fonctions de Matrix ont donc été utilisées et redimensionnées avec du 
développement par MNM Consulting et le soutien de la DSI de Valeo pour matérialiser les roadmaps. Le choix de ce SI s'explique par le fait qu'il était déjà présent dans l'entreprise, qu'il avait fait ses preuves sur des fonctionnalités et que les coûts des licences étaient donc déjà amortis en grande partie par les autres applications au sein de l'entreprise.

Figure 3 - Représentation simplifiée de l'architecture du SI des roadmaps de management

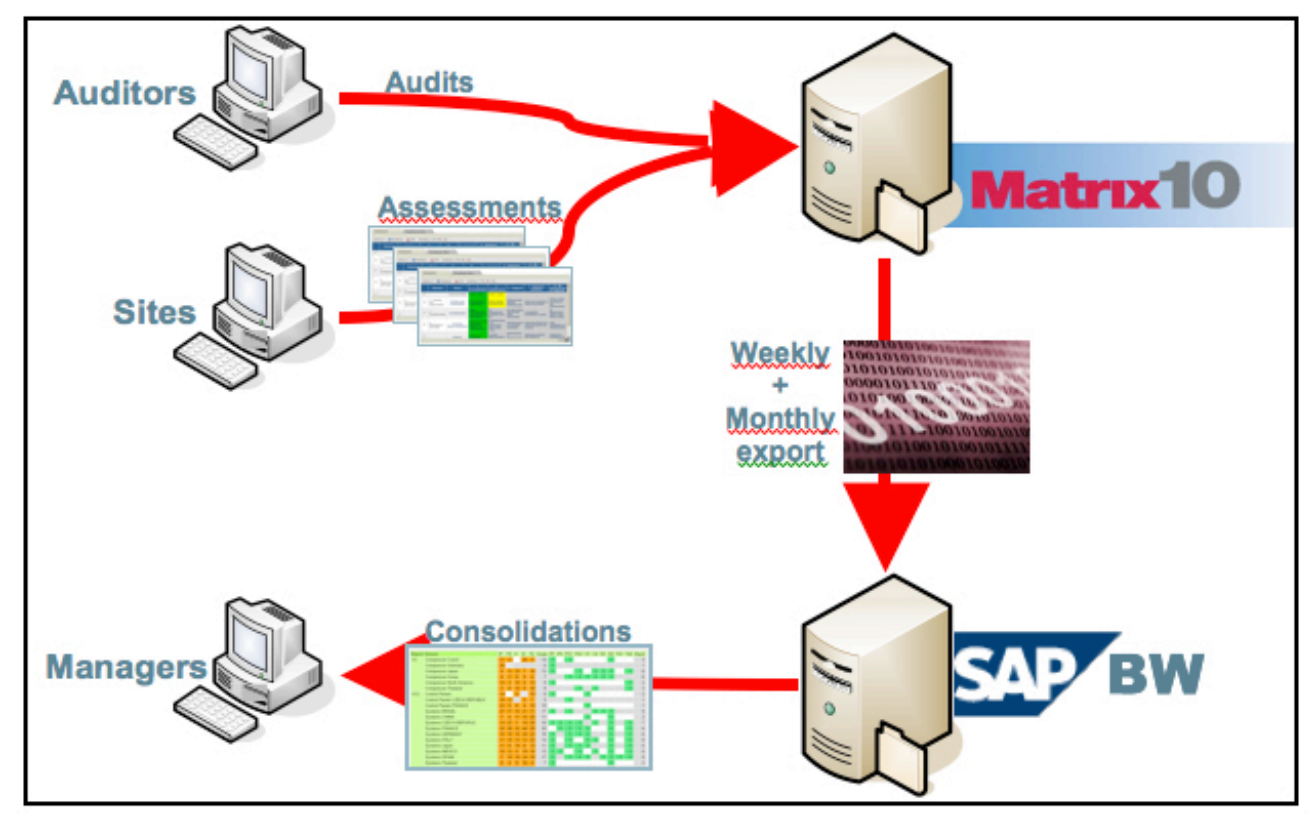

Source : MNM/Valeo

Afin de faciliter l'appropriation des roadmaps de management à partir de Matrix, le cabinet de conseil a élaboré avec l'équipementier une interface de type web pour que les utilisateurs puissent avoir un accès distant et alimenter facilement l'outil en données. Certaines fonctions transversales ont également été ajoutées pour permettre aux utilisateurs d'échanger des informations ou de poser des questions (FAQ, forums locaux...). En parallèle, une formation en ligne (dans un format visioconférence) de deux heures a été mise en place et animée par un des consultants de MNM et un manuel d'utilisateur a été diffusé en format électronique à l'ensemble des participants. La traduction des roadmaps sur Matrix (cf. figure 4) est donc matérialisée par une interface web qui peut être consultée à distance et qui masque pour les utilisateurs la plateforme informatique d'origine. On peut constater d'après la figure ci-dessous que la représentation des roadmaps de management est très proche d'un visuel de type tableau de bord de type reporting accentuant la dimension contrôle du SI, notamment au niveau de la perception des utilisateurs, sachant que ces données sont consolidées ensuite pour la Direction Générale de Valeo. 
Figure 4 - Exemple d'une roadmap de management dans la plateforme Matrix

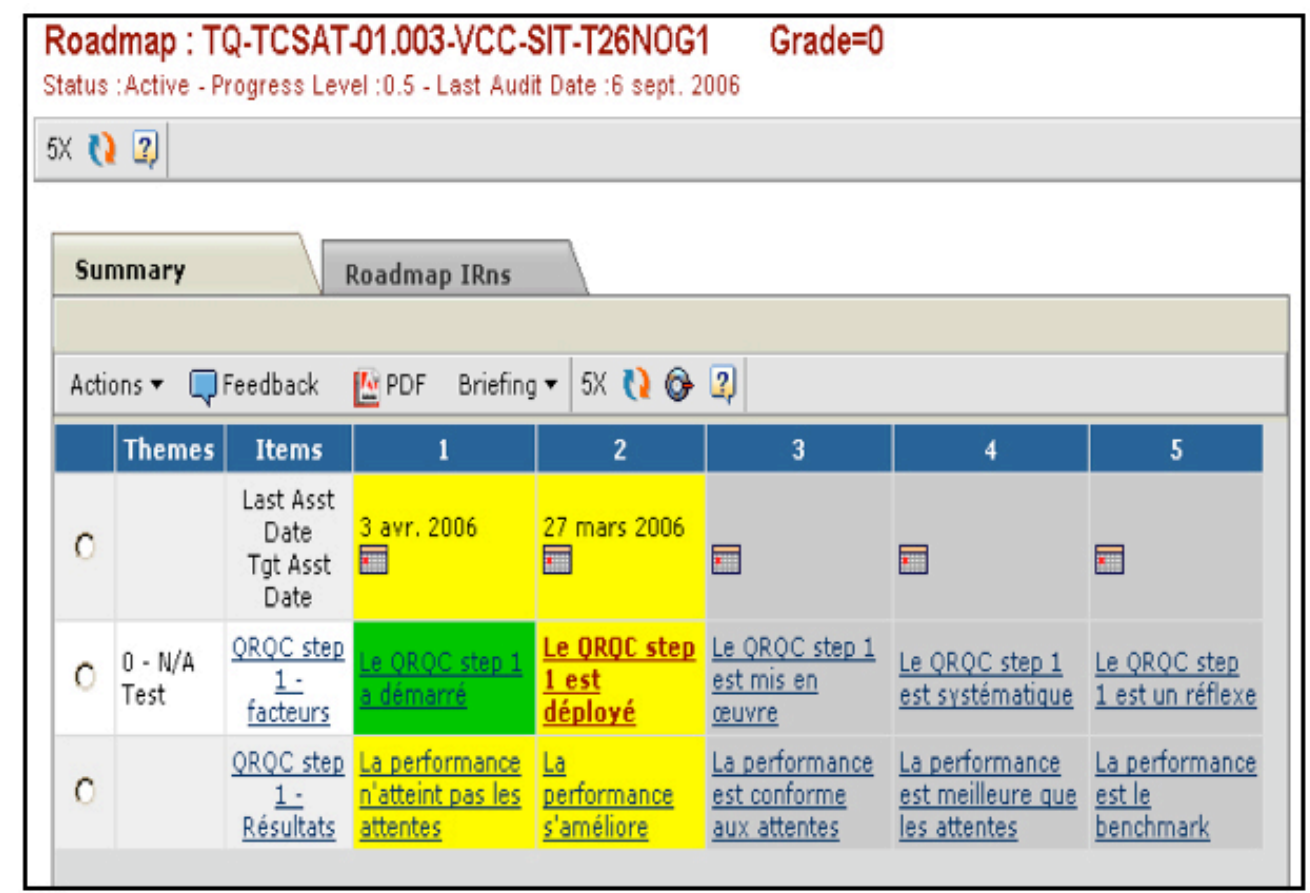

Source: VRM User Manual (V2)

Le tableau ci-dessous synthétise les apports de Matrix aux roadmaps de management. Il permet de constater que l'outil de gestion est bien transposé dans le SI et qu'il concerne l'ensemble des dimensions évoquées dans la première partie (contrôle, coordination et pilotage). Les fonctionnalités offertes par le SI ont permis l'extension des roadmaps de management à l'ensemble des entités sachant par ailleurs que des responsables d'axes ont été nommés par l'entreprise.

Tableau 2 - Les apports de Matrix aux roadmaps de management

\begin{tabular}{|c|c|c|}
\hline & Les Roadmaps de Management (RM) & Le SI (Matrix) \\
\hline Contrôle & $\begin{array}{l}\text { Chaque niveau atteint doit faire } \\
\text { l'objet de délivrables au niveau des } \\
\text { RM } \\
\text { Logique d'auto-assessment sur les } \\
\text { sites avec un audit une fois par an }\end{array}$ & $\begin{array}{l}\text { Les délivrables sont souvent des } \\
\text { documents informatiques stockés sur } \\
\text { la plateforme } \\
\text { Capitalisation et mémorisation des } \\
\text { niveaux atteints } \\
\text { Possibilité de tracer les événements } \\
\text { Les responsables de RM remplissent } \\
\text { eux même le SI directement } \\
\text { Les auditeurs doivent valider l'auto- } \\
\text { évaluation des sites via les RM }\end{array}$ \\
\hline Coordination & $\begin{array}{l}\text { Déclinaison des axes stratégiques } \\
\text { dans les RM (approche normative) } \\
\text { Les RM doivent permettre un } \\
\text { alignement des niveaux de maturité } \\
\text { de sites similaires }\end{array}$ & $\begin{array}{l}\text { Déploiement sur tous les sites des } \\
\text { RM à partir d'un accès Internet } \\
\text { Etat des sites en quasi-temps réel au } \\
\text { niveau des indicateurs complétés } \\
\text { Chaque entité peut à partir du SI voir } \\
\text { certaines RM selon les droits définis, } \\
\text { ainsi que communiquer avec d'autres } \\
\text { sites via les outils en ligne (outil de } \\
\text { feedback sur les livrables également) }\end{array}$ \\
\hline Pilotage & $\begin{array}{l}\text { Progression sur } 5 \text { niveaux des sites } \\
\text { de l'entreprise } \\
\text { Idée de passage du best effort à un } \\
\text { travail structuré (référentiel commun) }\end{array}$ & $\begin{array}{l}\text { Reporting des niveaux de maturité } \\
\text { consolidés par des tableaux de bord } \\
\text { au niveau de la Direction } \\
\text { Formalisation de l'outil de gestion au } \\
\text { travers de Matrix avec une interface } \\
\text { commune et en anglais }\end{array}$ \\
\hline
\end{tabular}




\section{3- Caractéristiques du SI et déploiement de l'outil de gestion}

Les roadmaps de management représentent un enjeu stratégique pour le pilotage des plans de progrès et l'alignement des différents sites géographiques sur les meilleures pratiques. Valeo est une illustration d'une entreprise étendue et de la problématique de généralisation des outils de gestion à l'ensemble des entités pour permettre un déploiement de la stratégie à l'ensemble de l'organisation. Nous allons donc analyser comment les caractéristiques de Matrix (le SI) ont facilité le déploiement des roadmaps de management et nous présenterons une discussion de notre cas pour voir comment le SI s'inscrit plus largement dans le dispositif de gestion des organisations.

\subsection{Les caractéristiques de matrix et leurs apports aux roadmaps de management}

Les roadmaps de management sont la conjugaison d'une méthode de management (pilotage de progrès par la déclinaison de la stratégie à travers un système d'indicateurs et l'organisation $\mathrm{du}$ reporting autour de ces indicateurs) et d'un outil informatique qui est une plateforme informatique basée sur des technologies web. L'outil de gestion s'appuie sur certaines propriétés intrinsèques du SI, notamment les trois fonctions analysées dans la première partie. Concernant la fonction de contrôle des roadmaps de management, on constate que la médiation de l'outil au travers d'une interface web via Matrix a contribué à clarifier les indicateurs d'une part avec l'écran comme interface, et, d'autre part, à apporter une visibilité des sites les uns par rapport aux autres, du moins pour les managers et les auditeurs. Gérer c'est moins organiser l'information entre les individus que «l'écran d'information » qui permet aux individus de se trouver dans une position de repérage stable par rapport à l'environnement (Gomez, 2006). Un responsable axe qualité suggérait même d'aller encore plus loin en « développant l'aspect graphique des indicateurs 5 axes pour un axe donné pour mettre en exergue les assessment et les résultats. Ceci permettra de voir s'il y a du window dressing ou pas » comme le montre la figure ci-dessous ou de montrer des écarts de performance à la moyenne qui peuvent être le résultat de pratiques innovantes. 


\section{Identification of roadmap modelling and deployment problems}

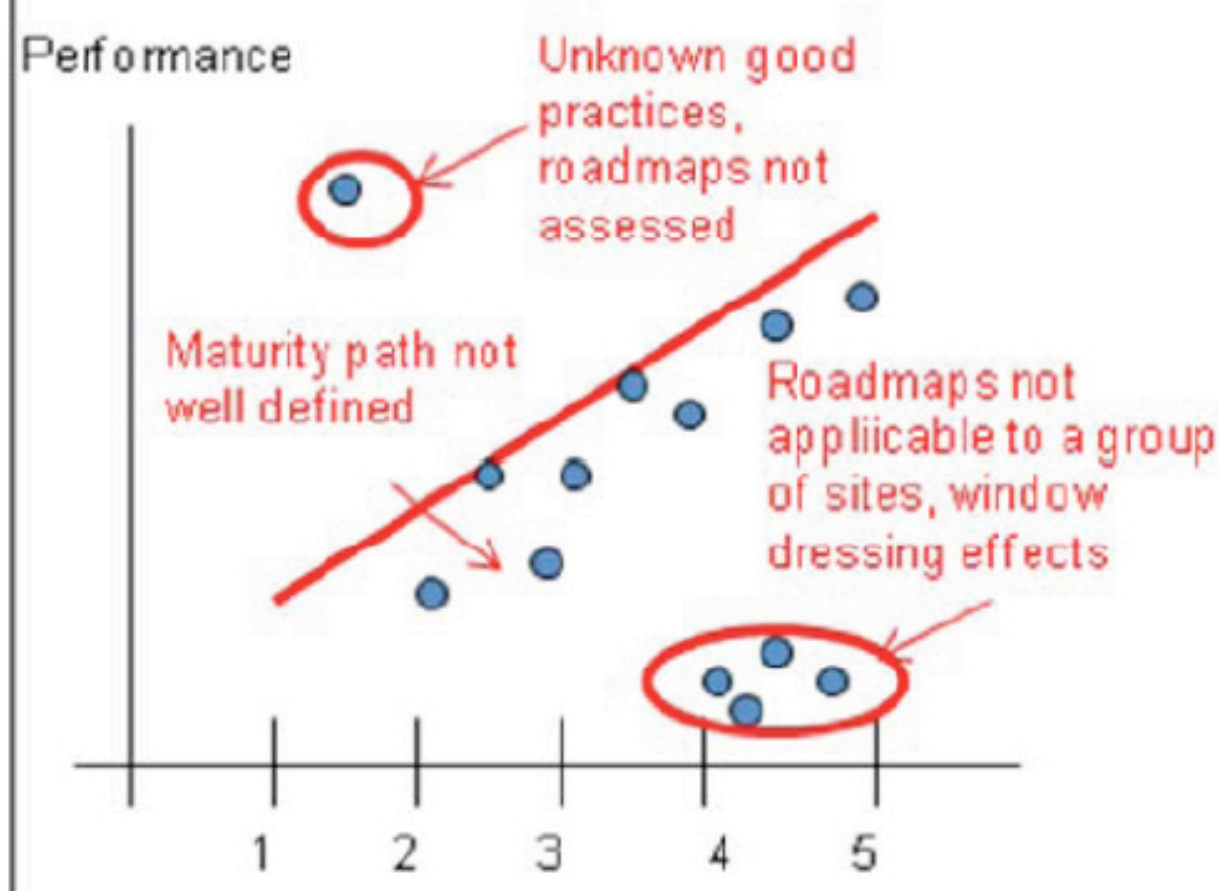

Source : Valeo/MNM

Comme le soulignent Aggeri et Labatut (2010, p. 18), « un agent ne se sert à un moment donné que d'un très petit nombre d'informations en privilégiant des paramètres numériques, parmi lesquels il privilégie ceux sur lesquels il se sent jugé et règle logiquement ses choix de manière à optimiser les jugements dont il se sent l'objet ». Les indicateurs et délivrables choisis pour passer d'un niveau à un autre contribuent à l'uniformisation des capacités organisationnelles via les responsables de roadmaps de management. Toutefois, cette formalisation de l'activité organisée au sens de Moisdon (1997) au sein d'une interface graphique et contextuelle n'est pas sans poser de questions car elle met en exergue le degré de granularité optimal et l'interprétation des données par les acteurs. L'un des rédacteurs d'axe soulignait ainsi « la difficulté de trouver le juste milieu entre la roadmap générale et la roadmap trop précise ». Enfin, la dimension contractuelle des objectifs définis via les roadmaps de management est présente dans les objectifs individuels des managers et responsables d'axes mais l'objectif est également, et c'est ce qui est nouveau, d'identifier des entités « hors normes » car elles ont pu innover en termes de pratiques.

L'autre aspect concerne la fonction de coordination. L'instrumentation constitue une forme privilégiée d'intervention pour construire de nouvelles capacités d'action (Joas, 1992) et participe à la transformation des activités et des organisations. Dans ce cadre, les roadmaps sont médiatisées via Matrix ce qui permet une consolidation multi-sites en quasi temps réel et donc in fine un pilotage de la stratégie à partir d'un état le plus à jour et le plus précis possible. Les propriétés du SI en matière de circulation et de consolidation des données permettent de modéliser les capacités de l'organisation et de répartir les ressources. C'est une des raisons pour lesquelles le déploiement des roadmaps a été porté par la Direction Générale de Valeo et s'est imposé rapidement dans l'organisation. Par ailleurs, le SI actuel permet des échanges intra-organisationnels entre les différentes entités par le biais notamment de 
forums et surtout de la possibilité de consulter l'ensemble des roadmaps disponibles selon les droits d'accès. Un ingénieur opérationnel indiquait ainsi que « la mise en place des roadmaps incite à communiquer et échanger avec d'autres entités : une fois tous les deux mois, vous rencontrez des sites à proximité pour échanger sur vos bonnes pratiques, les roadmaps ont créé ce besoin car il y a des steps que vous ne comprenez pas, les échanges permettent de savoir comment les interpréter ». On peut néanmoins se demander si les roadmaps ne génèrent pas de la complexité intrinsèquement qui peut être accentuée par le SI (ergonomie, traitement d'un plus grand nombre de données et d'indicateurs, création de métadonnées...). Les roadmaps de management ont également une fonction de capitalisation des meilleures pratiques ${ }^{7}$. Matrix, en tant que SI, permet de conserver les roadmaps de management et les délivrables en format électronique. Il s'apparente alors à un espace de stockage accessible à l'ensemble des utilisateurs qui peuvent accéder aux différentes données et trouver des solutions aux problèmes rencontrés.

Le dernier point concerne le pilotage de la stratégie et son opérationnalisation dans les différentes entités sachant que la Direction Générale s'est beaucoup impliquée dans la conception et l'amélioration des roadmaps de management. La consolidation des données de chaque entité en quasi-temps réel représente un appui pour le pilotage de la stratégie de Valeo. Cette logique, encore très top-down, doit évoluer vers une logique bottom-up avec les nouvelles fonctionnalités du SI qui permettront plus d'échanges et une meilleure opérationnalisation des décisions stratégiques. Un des rédacteurs d'axe considère ainsi que «l'outil doit permettre de faire des plans d'action et de les suivre, de faire des comparaisons par rapport aux objectifs. L'outil doit permettre d'écrire des commentaires et offrir des possibilités de modifications » et un ingénieur opérationnel achat cite « elles permettent une meilleure communication et du progrès dans la pratique, amenant sur le long terme de la performance ». L'un des objectifs en cours de développement est l'ajout de fonctionnalités de type Web 2.0 (chats, forums, espaces collaboratifs, gestion des communautés...) afin de faire en sorte que les roadmaps de management soient moins perçues comme des outils de reporting. Dans cette optique, le SI apporte ses propriétés intrinsèques permettant de faire évoluer le rôle de l'outil de gestion vers une dimension plus personnelle et individuelle pour les acteurs (personnalisation de l'interface notamment). Lors de l'un de nos entretiens avec un responsable de Valeo, ce dernier soulignait que la sphère informatique individuelle peut servir de porte d'entrée pour l'utilisation des applications collaboratives et de reporting qui sont dans une sphère institutionnelle. Enfin, la plateforme informatique a également contribué à normer les comportements des acteurs via les indicateurs choisis et les délivrables associés. Elle permet d'orienter les actions des entités. De l'échange d'information on passe à l'observation de l'écran que constituent les règles afin de procurer un système d'interprétation de l'information (Gomez, 2006), notamment à partir du SI dans notre cas. La visualisation des indicateurs, notamment de manière relative par rapport aux autres entités, peut jouer comme un levier et une incitation à progresser pour les entités utilisant les mêmes roadmaps de management. Cela est accentué par la culture de Valeo où « pour progresser, il faut mesurer» (DSI de Valeo).

\subsection{Discussion du cas}

Les roadmaps ne font sens qu'à partir du moment où elles sont généralisées dans l'ensemble de l'organisation. L'enrichissement des fonctionnalités à partir du SI confère une dimension extensive aux roadmaps de management, que ce soit au niveau de l'utilisateur ou des fonctionnalités propres au contrôle, à la coordination et au pilotage de la stratégie. Le choix de Matrix, pour supporter les roadmaps de management, a permis de s'assurer d'un déploiement à grande échelle avec un SI robuste et fiable. Matrix a été qualifié par l'un des consultants du cabinet de conseil comme « un outil à la russe », à savoir un outil éprouvé mais relativement peu modulable et évolutif. Toutefois, la transposition de ce SI dans une interface de type web permet une diffusion des roadmaps à l'ensemble des entités de l'entreprise. Cette première condition s'explique par l'enjeu stratégique des roadmaps de management et par une logique de réduction des coûts. 
Dans le cas de Valeo, le SI et l'outil de gestion sont complètement superposés au niveau des utilisateurs. Ce dernier prend forme dans le SI qui, en plus de le matérialiser avec une interface visuelle, rend possible son déploiement sur l'ensemble des sites à la condition que ces derniers possèdent un accès Internet fiable et rapide, ce qui n'est pas toujours le cas selon les zones géographiques. Les 5 axes de progrès définis par la Direction Générale de l'entreprise se déclinent ainsi sur près de 17000 roadmaps de management. Le SI donne ainsi vie aux roadmaps de management et il apparaît difficilement concevable qu'elles existent hors de la plateforme, du moins à cette échelle de diffusion. Bien qu'il soit possible de formaliser les roadmaps de management sur des fichiers Excel, les expériences réalisées par quelques utilisateurs réfractaires au SI ne sont montrées peu concluantes sans la présence d'une plateforme informatique. Enfin, le discours dans la démarche de prospection de nouveaux clients est plus axé sur la plateforme informatique (Roadmap Manager) que sur la méthode en tant que telle. On pourrait dire que le SI fait corps avec l'outil et la méthode de gestion.

Notre étude de cas montre que le SI de Valeo représente le substrat technique de l'outil de gestion (Hatchuel et Weil, 1992) qui doit être matérialisé pour prendre vie dans l'organisation. Quelques travaux suggèrent ainsi que les objets de gestion informatisés s'inscrivent aujourd'hui de plus en plus dans des outils de gestion, voire des dispositifs de gestion (Carton et al., 2006). Notre cas illustre également que les SI sont un élément du dispositif de gestion ${ }^{8}$ dans les organisations et qu'ils peuvent être superposés aux outils de gestion selon les configurations organisationnelles et les cultures d'entreprise.

Figure 6 - Schéma intégrateur du dispositif de gestion chez Valeo

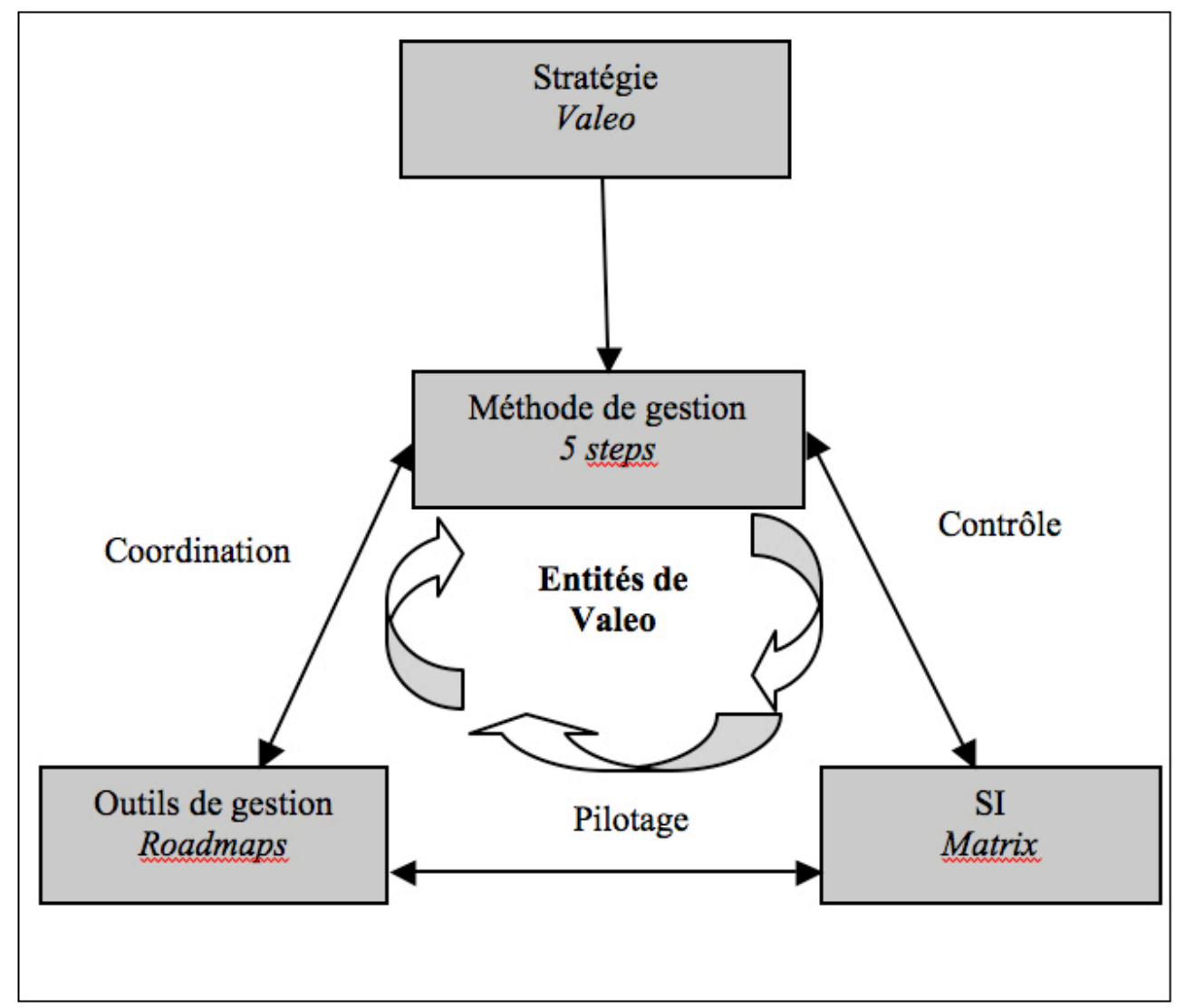

Le cas de Valeo confirme également le besoin d'un alignement stratégique entre les dimensions stratégiques, organisationnelles et technologiques (Henderson et Venkatraman, 1993). L'une des conséquences est qu'intervenir sur le SI revient à modifier l'organisation (David et Pallez, 2001). Notre étude de cas sur Valeo confirme bien que le SI n'est pas un objet neutre dans la diffusion des outils de gestion et qu'il va interférer et, pas seulement en tant qu'infrastructure technique. La médiation totale ou partielle de l'outil de gestion au sein du SI lui confère une dimension extensive et semi-finie compte tenu des nouvelles fonctionnalités 
offertes. Le pilotage ne doit plus être subi par les opérationnels et le changement ne doit plus être perçu comme des exigences issues de la direction générale et déconnectées des réalités du terrain (Brignano, 2006). Valeo a néanmoins compris qu'il ne suffisait pas de mettre en place un outil de gestion pour évoluer vers un management plus participatif : c'est là où le SI et l'interface Internet font sens pour ajouter des fonctionnalités (forums, plateforme wiki, réseau social...) qui servent plus aux entités qu'à la direction de l'entreprise, tout en assurant la remontée et la prise en compte d'informations terrain pour un pilotage communautaire avec rétroactions (vision « $2.0 »)$. Des fonctions de feedbacks ont déjà été généralisées sur la plateforme informatique à l'ensemble des délivrables et des projets d'applications plus centrées sur la sphère individuelle sont en cours de développement. Ces fonctionnalités de communication propres au SI devraient faciliter l'appropriation par les utilisateurs de l'outil qui deviendrait plus intuitif et adapté à chaque situation des entités. Autrement dit, le SI devrait permettre une instanciation des situations avec une personnalisation de l'interface selon les besoins tout en conservant les indicateurs nécessaires au pilotage.

Zoom sur le déploiement des applications $2.0 \mathrm{chez}$ Valeo

La sphère de travail en équipe était la moins pourvue chez Valeo en termes d'applications et représentait un cadre idéal de développement d'applications pour favoriser la collaboration. De plus, l'ancien système n'avait pas atteint le niveau de performance attendu dans la logique bottom-up et il y avait un besoin croissant en interne de plus de décloisonnement et de collaboration entre les différents services qui fonctionnaient encore en silos. Il est intéressant de noter que cela était aussi une demande des équipes de disposer d'outils collaboratifs. Le déploiement des applications s'est réalisé sur un principe « de découverte » pour les utilisateurs en partant du principe que l'adoption se fait par les usages. Si les applications créent de la valeur ajoutée et sont perçues comme «problem solvers », elles vont se propager naturellement dans l'organisation par les acteurs internes. Les applications du Web 2.0 fonctionnent aussi beaucoup sur le principe du « self service » (exemple des Google sites chez Valeo qui ont été mis à disposition des acteurs et qui n'ont pas été imposés). Toutefois la dynamique de diffusion, même avec le Web 2.0, ne peut pas concerner tous les individus de la même manière. Il est important de « respecter les rythmes des individus » dans l'adoption des applications. La DSI a ainsi adopté une posture de propositions (sauf à certains moments clés dans le projet) car il est toujours préférable que les services viennent spontanément la solliciter. En interne, la notion de «stratégie 2.0 » a été utilisée pour signifier l'importance de ces applications et de leur intégration dans les processus.

\section{Source : entretien interne chez Valeo}

Comme le montre l'encadré ci-dessus, le SI devient lui aussi porteur d'une philosophie gestionnaire avec ses propres fonctionnalités et les propriétés de l'outil de gestion qu'il supporte. Dans notre exemple de l'ajout de fonctionnalités en Web 2.0, c'est bien la recherche d'un échange productif qui est souhaité par l'entreprise à partir d'une structuration spécifique du SI pour accueillir ces activités discursives tout en incluant une forme d'intéressement (Detchessahar, 2003). Un autre exemple simple relève de la gestion des droits d'accès via la plateforme informatique aux roadmaps de management qui peut faire varier le degré de transparence et d'accès à l'information selon les organisations. Cela questionne également la capacité de l'entreprise à intégrer dans son infrastructure existante le SI en plus de la conduite du changement nécessaire pour l'outil de gestion, sachant que les problématiques sont différentes entre les aspects techniques et managériaux. La réussite de l'outil de gestion repose également sur le niveau d'appropriation du SI par les utilisateurs et le management intermédiaire. C'est donc une donnée supplémentaire à prendre en compte dans le choix 
des outils de gestion, au même titre que l'interopérabilité avec les SI déjà présents dans l'organisation.

\section{Conclusion}

Les outils de gestion se matérialisent de plus en plus dans les SI et profitent des propriétés de ces derniers pour mieux s'intégrer dans les organisations. Les propriétés du SI de Valeo illustrent la dimension extensive conférée aux roadmaps de management. Notre étude de cas sur Valeo illustre la superposition des roadmaps de management et de la plateforme Matrix, pas uniquement au niveau de l'infrastructure technique. On peut même affirmer que SI est devenu aussi important que l'outil et la méthode de gestion dans une dimension intégrée. Sur le plan managérial, cette médiatisation des outils de gestion conduit d'après notre recherche à prendre en compte beaucoup plus en amont les conditions d'appropriation et d'utilisation des SI pour arriver au niveau de performance souhaité et aux objectifs stratégiques. La question est encore plus accentuée dans les organisations étendues du fait de la complexité du pilotage et du déploiement de la stratégie. Notre étude de cas soulève également la question centrale de la conception des outils de gestion et des SI. Comme nous l'avons rappelé en introduction, ces derniers soutiennent de plus en plus souvent le système de gestion des entreprises. On peut donc légitimement s'interroger sur la pertinence d'une logique de co-conception simultanée des deux objets (SI et outils de gestion) ? Est-il nécessaire de s'interroger sur les propriétés des SI supports lorsqu' on élabore un outil de gestion ou de pilotage sachant que les deux objets ont des propriétés communes parfois, même indissociables (accessibilité aux informations, centralisation/décentralisation...) ? Faut-il prendre en compte les SI déjà existants dans les organisations dans une logique de dépendance du sentier et d'effets d'apprentissage ? Etc. Ces questions nous ramènent aux méthodes de conception à mettre en œuvre, mais également aux compétences et connaissances nécessaires qui sont pour l'instant détenues par plusieurs acteurs distincts qui doivent se coordonner dans les processus de conception et de déploiement des outils de gestion (DSI de l'entreprise, cabinets de conseils, SSII, managers, comité de direction...). Nous avons également observé dans notre cas que les réflexions sur le Web 2.0 favorisent l'évolution de la DSI vers «moins de techniques et plus de fonctionnel », ce qui est une tendance forte depuis plusieurs années. La mission de la DSI chez Valeo est ainsi «d'accompagner la collaboration dans l'organisation ». L'une des évolutions possibles serait de voir les DSI comme des co-architectes de l'organisation, au même titre qu'ils sont en charge de l'urbanisation des SI.

34 Notre recherche contribue à montrer sous quelles conditions le SI peut être vu comme un mécanisme causal de transformation des organisations (Besson et Rowe, 2011). Sur le plan conceptuel, l'un des apports de ce cas est d'illustrer la transformation organisationnelle en soulignant que le processus de transformation s'établit avec un niveau intermédiaire mais essentiel, celui des outils de gestion. Ce niveau permet de mieux comprendre comment peut évoluer le poids des différentes dimensions (contrôle, coordination et stratégie) selon l'extension de l'outil de gestion à partir des propriétés et des fonctionnalités du SI. Cette recherche souligne également que le SI doit être intégré dans le dispositif de gestion car il permet l'existence même de l'outil de gestion bien que ce dernier supporte également les contraintes de la plateforme (connexion, ergonomie...). Par ailleurs, les philosophies gestionnaires sous-jacente aux outils de gestion et aux SI (logique open source ou propriétaire, degré de personnalisation de l'interface par l'utilisateur, degré d'accessibilité...) doivent également converger compte-tenu de l'interdépendance soulevée par ces deux éléments dans le dispositif de gestion. Si l'on prolonge la logique, le SI deviendrait un objet de transformation de l'organisation et contribuerait alors au design des organisations. Notre cas souligne le caractère co-évolutif du SI et de l'organisation.

Toutefois, notre recherche doit être approfondie car elle présente plusieurs limites, dont notamment celle de la validité externe. Le terrain repose sur un seul cas, celui de Valeo, et pose la question de la généralisation de nos résultats. Nous avons cherché au cours de cette recherche une généralisation analytique (Ayerbe et Missonier, 2007) en mettant en correspondance des éléments empiriques et théoriques par des itérations successives. Toutefois, notre grille de 
lecture pourrait être reprise dans d'autres contextes pour vérifier l'articulation entre le SI et l'outil de gestion. Les schémas d'articulation entre les SI, les outils de gestion et l'organisation pourraient faire également l'objet d'approfondissements avec une analyse plus précise des facteurs de contingence des organisations. Enfin, une relecture des travaux sur l'appropriation par les acteurs des SI sous le prisme des outils de gestion pourrait être menée pour apporter un éclairage complémentaire.

Yin R.K. (2003), Case Study Research: Design and Methods, Sage

Publications.

Nous tenons à remercier Nicolas Monomakoff (dirigeant de MNM) et François Blanc (DSI de Valeo) pour leur disponibilité, ainsi que Ibrahima Fall et Philippe Rauffet pour l'accès à leur matériau de recherche et aux nombreux échanges que nous avons pu avoir avec eux.

\section{Bibliographie}

Aggeri F. et Labatut J. (2010), « La gestion au prisme de ses instruments. Une analyse généalogique des approches théoriques fondées sur les instruments de gestion », Finance Contrôle Stratégie, vol. 13, $\mathrm{n}^{\circ} 3$, p. 5-37.

Amabille S. et Gadille M, (2006), « Coopération interentreprises, système d'information et attention organisationnelle », Revue Française de Gestion, $\mathrm{n}^{\circ}$ 164, p. 97-118.

Argyres N. (1999), « The Impact of IT on Coordination: Evidences From the B-2 'Stealth' Bomber », Organization Science, vol. 10, $\mathrm{n}^{\circ}$ 2, March-April, p. 162-180.

Ayerbe C. et Missonier A. (2007), «Validité interne et validité externe de l'étude de cas : principes et mise en oeuvre de l'étude de cas », Finance Contrôle Stratégie, vol. 10, n 2, p. 37-62.

Barrett S. et Konsynski B. (1982), «Inter-Organization Information Sharing Systems », MIS Quarterly, vol. $6, n^{\circ} 4$, p. 93-104.

Bernasconi M. (1996), « Les systèmes d'information interorganisationnels sont-ils toujours source d'avantages concurrentiels durables ? », Systèmes d'Information et Management, vol. 1, n 1, p 7-25.

Besson P. et Rowe, F. (2011), «Perspectives sur le phénomène de la transformation organisationnelle », Systèmes d'Information Management, vol. 16, $\mathrm{n}^{\circ}$ 1, p. 3-34.

Blanc F. et Monomakhoff N. (2008), La méthode 5 steps, Editions AFNOR.

Benghozi P.J. (2001), « Technologies de l'information et organisation : de la tentation à la flexibilité à la centralisation », Gestion 2000, ${ }^{\circ} 2$, mars-avril, p. 61-80.

Brignano S. (2006), Communication RH quelles réalités ?, entreprises et carrières, éditions liaisons.

Carton S., De Vaujany F.X. et Perez M. (2006), «Vers une théorie de l'appropriation des outils de gestion informatisés : une approche intégrative », Management et Avenir, n 9, p. 159-179.

Chandler A. (1962), Strategy and structure, MIT Press, Cambridge, USA.

Clemons E.K. et Row M.C. (1991), « Sustaining IT advantage: The role of structural” differences », MIS Quarterly, vol. 15, n 3, p. 275-292.

David A. et Pallez F. (2001), « Les systèmes d'information à l'épreuve de l'organisation », Ingénierie des systèmes d'information, in C. Cauvet et C. Rosenthal Sabroux, Hermès, p. 23-60.

David A. (1999), «Logique, épistémologie et méthodologie en sciences de gestion », colloque de l'AIMS.

De Corbière F. (2011), «SIIO et amélioration de la qualité des données : analyse de la synchronisation des données par catalogues électroniques », Systèmes d'Information et Management, vol. 16, n 3, p. 45-79.

De Vaujany F.X. (2010), « Pour une théorie de l'appropriation des outils de gestion : vers un dépassement de l'opposition conception-usage », Revue Management et Avenir, n 9, p. 109-126.

De Vaujany F.X. (2005), De la conception à l'usage : vers un management de l'appropriation des outils de gestion, éditions EMS.

Detchessahar M. (2003), « L'avènement de l'entreprise communicationnelle », Revue Française de Gestion, $\mathrm{n}^{\circ} 142$, p. 65-84.

DiMaggio P.J. et Powell W.W. (1983), « The Iron Cage Revisited: Institutional Isomorphism and Collective Rationality in Organizational Fields », American Sociological Review, vol. 48, p. 147-160. 
Eisenhardt K.M. (1989), « Building Theories From Case Study Research », Academy of Management Review, vol. $14, \mathrm{n}^{\circ} 4$, p. 532-550.

Gomez P.Y. (2006), «Information et conventions : le cadre du modèle général », Revue Française de Gestion, $\mathrm{n}^{\circ}$ 60, p. 217-240.

Hagel A. (1996), "Spider vs Spider", The McKinsey Quarterly, ${ }^{\circ} 1$.

Hatchuel A. et Weil B. (1992), L'expert et le système, Economica.

Henderson J.C. et Venkatraman N. (1993), « Strategic Alignment: Leveraging Information Technology for Transforming Organizations », IBM Systems Journal, vol. 32, n 1, p. 4-16.

Jarillo C. (1993), Strategic Networks - Creating Borderless Organization, ButterWorth Heinemann.

Joas H. (1992), La créativité de l'agir, Le Cerf.

Kettinger W.J., Grover V., Guha S. et Segars A.H. (1994), "Strategic Information Systems Revisited: A Study in Sustainability and Performance", MIS Quarterly, vol. 18, n 1, p. 31-58.

Konsynski B.R. (1993), "Strategic Control In The Extended Enterprise," IBM Systems Journal, vol. 32, $\mathrm{n}^{\circ} 1$, p. 111-142.

Le Moigne J.L. (1977), La théorie du système général, Théorie de la modélisation, Paris : Col., SystèmesDécisions, Presses Universitaires de France.

Lorino, P. et Teulier, P. (2005), « Des connaissances à l'organisation par l'agir collectif », Entre connaissance et organisation : l'activité collective, La Découverte, p. 11-25.

Markus M.L. (2010), "On The Usage of Information Technology: The History of IT and Organization Design in Large US Enterprises”, Entreprises et histoire, n ${ }^{\circ}$ 60, p. 17-28.

Moisdon J.C. (1997), Du mode d'existence des outils de gestion, Editions Seli Arsan, Paris.

Naulleau G. et Guth J.P. (2000), « Du partenariat à l'entreprise étendue, vers une reconfiguration de la relation client/fournisseurs dans le secteur automobile », Gérer et Comprendre, n ${ }^{\circ}$ 61, p. 31-41.

Ouchi W.G. (1979), "A Conceptual Framework for the Design of Organizational Control Mechanisms", Management Science, vol. 25, $\mathrm{n}^{\circ}$ 9, p. 833-848.

Porter M. et Millar V. (1985), « How Information Technology Gives You A Competitive Advantage », Harvard Business Review, vol. 63, n 4, p. 149-160.

Rasmussen J., Brehmer B. et Leplat J. (1991), Distributed Decision Making: Cognitive Models For Cooperative Work, Wiley.

Richardson G.B. (1972), “The Organisation of Industry”, Economic Journal, vol. 82, n 327, p. 883-896.

Reix R. (1999), «Les technologies de l'information, facteurs de flexibilité ? », Revue Française de Gestion, n $^{\circ} 123$, p. 111-119.

Thorelli H.B. (1986), « Networks Between Markets and Hierarchies », Strategic Management Journal, vol. 7, p. 37-51.

Williamson O.E. (1985), The Economic Institutions of Capitalism. Firms, Markets, Relational Contracting, Free Press.

\section{Annexe}

Liste des personnes interrogées et des entretiens menés chez MNM

\begin{tabular}{|l|l|l|}
\hline Prénom & Fonction & $\begin{array}{l}\text { Thèmes principaux abordés lors des } \\
\text { entretiens et réunions de travail }\end{array}$ \\
\hline Nicolas M & Dirigeant de MNM & $\begin{array}{l}\text { Roadmaps de management, Matrix, } \\
\text { organisation de Valeo, 5 steps }\end{array}$ \\
\hline Michel L & Développeur de MNM & $\begin{array}{l}\text { Roadmaps de management, Matrix, } \\
\text { organisation de Valeo, SI, Web 2.0 }\end{array}$ \\
\hline Roland A & Chef de projet et formation de MNM & $\begin{array}{l}\text { Roadmaps de management, Matrix, } \\
\text { organisation de Valeo, formation chez V }\end{array}$ \\
\hline Nicolas A & Directeur technique de MNM & $\begin{array}{l}\text { Roadmaps de management, Matrix, SI, } \\
\text { Web 2.0 }\end{array}$ \\
\hline Hubert F & Développeur de MNM & $\begin{array}{l}\text { Roadmaps de management, Matrix, } \\
\text { organisation de Valeo, SI, Web 2.0 }\end{array}$ \\
\hline Ibrahima F & $\begin{array}{l}\text { Consultant de MNM et doctorant en } \\
\text { CIFRE }\end{array}$ & $\begin{array}{l}\text { Roadmaps de management, } \\
\text { organisation de Valeo, 5 steps, capacités } \\
\text { organisationnelles }\end{array}$ \\
\hline
\end{tabular}




\begin{tabular}{|l|l|l|}
\hline Philippe R & Doctorant en CIFRE & $\begin{array}{l}\text { Roadmaps de management, Matrix, SI, } \\
\text { Web 2.0 }\end{array}$ \\
\hline
\end{tabular}

Liste des entretiens menés par Philippe chez Valeo

\begin{tabular}{|l|l|l|l|l|}
\hline & Responsables d'axe & Rédacteurs & $\begin{array}{l}\text { Ingénieurs } \\
\text { Opérationnels }\end{array}$ & Auditeurs \\
\hline Bayen & 1 & 3 & 0 & 3 \\
\hline Valeo Service & 3 & 0 & 1 & 0 \\
\hline Valeo Transmissions & 3 & 0 & 0 & 0 \\
\hline Valeo Compressors & 1 & 0 & 0 & 0 \\
\hline Valeo Climate Control & 2 & 0 & 3 & 0 \\
\hline $\begin{array}{l}\text { Valeo Electrical } \\
\text { Systems }\end{array}$ & 1 & 0 & 0 & 0 \\
\hline $\begin{array}{l}\text { Nombre de personnes } \\
\text { interrogées }\end{array}$ & 11 & 3 & 4 & 2 \\
\hline
\end{tabular}

Liste des entretiens menés par Ibrahima chez Valeo

\begin{tabular}{|l|l|l|l|l|}
\hline & Responsables d'axe & Rédacteurs & $\begin{array}{l}\text { Ingénieurs } \\
\text { Opérationnels }\end{array}$ & Auditeurs \\
\hline $\begin{array}{l}\text { Nombre de personnes } \\
\text { interrogées }\end{array}$ & 8 & 3 & 4 & 3 \\
\hline
\end{tabular}

\section{Notes}

1 Balance Scorecard, méthode Activity Based Costing, Total Quality Management

2 « Ensemble des moyens (organisation, acteurs, procédures, systèmes informatiques) nécessaires au traitement et à l'exploitation des informations dans le cadre d'objectifs définis au niveau de la stratégie de l'établissement, des métiers, de la réglementation » (définition issue du glossaire du guide de conduite de projet systèmes d'information du CNRS et reprise par plusieurs cabinets de conseil et SSII). Nous intégrons dans le concept de SI, les Technologies de l'Information et de la Communication (TIC)

3 Signe du rapprochement des problématiques stratégiques, organisationnelles et informationnelles, la plupart des grands cabinets de conseil et de management ont désormais des compétences en interne en matière de SI, notamment par le biais des mouvements de rapprochement avec des SSII (Cap Gémini et Ernst \& Young, IBM et Price Waterhouse Coopers Consulting...).

4 L'entreprise étendue est une version optimale de l'entreprise vue comme un système. Sur le site Internet de Valeo, on peut trouver ainsi cette phrase : «L'entreprise étendue Valeo englobe ses employés, ses fournisseurs, ainsi que ses partenaires technologiques, tous travaillant pour le même objectif : la satisfaction des clients ».

5 Note : 5 Steps est le nom commercial de la méthode telle que présentée dans l'ouvrage « La méthode 5 Steps : pour déployer efficacement une stratégie », de F.Blanc, N.Monomakhoff, AFNOR Editions, avril 2008.

6 Product Lifecycle Management

7 Le taux de turnover chez Valeo est d'environ $10 \%$

8 Un dispositif de gestion est un ensemble d'éléments de design organisationnel porté par une intention stratégique, produit et géré par le centre ou le pivot d'un collectif organisé, et qui vise à intégrer les outils et les acteurs de façon cohérente, et dans le respect de certaines règles de gestion (De Vaujany, 2010)

\section{Pour citer cet article}

\section{Référence électronique}

Sébastien Tran, «Le pilotage des entreprises étendues : le rôle du SI dans le dispositif de gestion », Finance Contrôle Stratégie [En ligne], 15-1/2। 2012, mis en ligne le 02 août 2012, consulté le 25 novembre 2012. URL : http://fcs.revues.org/99 


\section{À propos de l'auteur}

\section{Sébastien Tran}

Ecole de Management de Normandie

9 rue Claude Bloch

14000 Caen

s.tran@em-normandie.fr

Chercheur associé à M-Lab (DRM - CNRS UMR 7088)

sebastien.tran@dauphine.fr

\section{Droits d'auteur}

Tous droits réservés

\section{Résumés}

Les systèmes de pilotage doivent assurer le déploiement de la stratégie dans l'ensemble de l'organisation dont le périmètre peut varier au gré des actions stratégiques. Ces systèmes reposent de plus en plus sur des outils de gestion mais peu répondent vraiment aux spécificités des nouvelles configurations comme les entreprises étendues ou se revendiquent comme permettant de les piloter. En parallèle, les Systèmes d'Information (SI) se sont diffusés massivement dans les organisations pour soutenir des processus relevant de plus en plus du pilotage des organisations et des outils de gestion. L'objet de cet article est de déterminer quelles sont les caractéristiques et le statut du SI dans le dispositif de gestion au sein d'une organisation de type étendu.

Steering systems must ensure the deployment of the strategy throughout the organization whose perimeters can vary according to strategic actions. These systems rely increasingly on management tools, but few truly meet the specific needs of new configurations as the extended organzation nor do they claim to allow them to be controlled. At the same time, Information Systems (IS) have spread massively through organizations to support processes which increasingly relate to the steering of organizations or management tools. The object of this article is to determine what role Information Systems can play in the management system within extended organizations.

\section{Entrées d'index}

Mots-clés : SI, stratégie, organisations étendues, outils de gestion

Keywords : IS, strategy, extended organizations, management tools 